\title{
A Modified Fractional Zener Model to Describe the Behaviour of a Carbon Fibre Reinforced Polymer
}

\author{
M. Fernanda P. Costa and C. Ribeiro \\ Department of Mathematics and Applications and Centre of Mathematics \\ University of Minho, Campus de Gualtar, 4710-057 Braga, Portugal
}

\begin{abstract}
In this work a modified conventional Fractional Zener Model is deduced and applied to estimate the viscoelastic constitutive parameters of a Carbon Fibre Reinforced Polymer. The accuracy of this modified model was studied against conventional Fractional Zener model and Fractional Maxwell model, considering experimental data in the frequency domain. The set of parameters was found by solving a nonlinear constrained least square problem based on the variation of the storage and loss moduli with frequency.
\end{abstract}

Keywords: inverse problem, nonlinear optimization, viscoelasticity, fractional derivative models

PACS: 02.60. Ed, 02.60. -x

\section{INTRODUCTION}

CFRP-Carbon Fibre Reinforced Polymer exhibit viscoelastic properties [1]. The combined effect of elastic and viscous behavior, intrinsic to the mechanical response of viscoelastic materials, may be described using differential constitutive stress-strain equations of zero and first order, respectively. The conventional stress-strain relations in theory of viscoelasticity found in the literature (such as Maxwell model, Kelvin-Voigt model, Zener model, PoyntingThompson model, etc..) have been determined by various combinations of springs and dashpots (in series or parallel). More recently, the fractional order viscoelasticity theory has been successfully employed by several authors (see e.g. [1], [2]) to describe the viscoelastic behavior of materials. The order of the fractional differential equations varies in the range $[0,1]$. The minimum order value correspond to the spring element while the maximum value correspond to the dashpot element. When an intermediate order is considered a new element called springpot, also called Blair model, appears capable of modelling the transition between a purely elastic and purely viscous behavior.

It is well established that the fractional constitutive models are more efficient than conventional integer-order models due to the fact that fewer parameters are required to represent the realistic viscoelastic material (see e.g. [3], [4], [5]). However, the Fractional Viscoelastic Models, also known as Generalized Viscoelastic Models, may feature different numbers of parameters and it is obviously that, depending on the number of parameters involved, some models should be more effective than others to accurately model specific mechanical characteristics of particular material types.

The main objective of this work is to study the influence of the number of parameters of fractional models, in the experimental data fitting accuracy. Thus, we propose a Modified Fractional Zener Model, considering $n$ springpots of fractional-order in parallel with a Fractional Maxwell Model ( 2 springpot elements in series). This way, the proposed Modified Fractional Zener Model (MFZM) involves $2 n+4$ parameters. To estimate the viscoelastic parameters of the MFZM, a nonlinear constrained least squares fit was considered. Then, we compare the viscoelastic response of a Carbon Fibre Reinforced Polymer modeled by the proposed MFZM and by the Fractional Maxwell Model (FMM) and Fractional Zener Model (FZM).

\section{A MODIFIED FRACTIONAL ZENER'S MODEL}

We introduce a fractional element called the springpot, which satisfies the following constitutive equation (see e.g. [2]):

$$
\sigma(t)=E \tau^{\alpha} D_{R L}^{\alpha} e(t)
$$


where $\sigma$ is the stress, $E$ the Young Modulus (elastic constant), $e$ the applied strain and $D_{R L}^{\alpha} f(t)$ is the fractional derivative of the Riemann-Liouville type of the order $\alpha$ of $f(t)$ with respect to time $t$, (see e.g. [1])

$$
D_{R L}^{\alpha} f(t)=\frac{1}{\Gamma(r-\alpha)} \frac{d^{r}}{d t^{r}} \int_{a}^{t}(t-\tau)^{r-\alpha-1} f(\tau) d t \tau(r-1<\alpha \leq, r \in \mathbf{N})
$$

where $\Gamma$ is the Euler Gamma function. In order to get a modified fractional viscoelastic model, we apply a FMM in parallel with $n$ springpot elements:

$$
\begin{gathered}
\sigma_{0}(t)+\tau_{0}^{\alpha_{1}-\alpha_{2}} D_{R L}^{\alpha_{1}-\alpha_{2}} \sigma_{0}(t)=E_{0} \tau_{0}^{\alpha_{1}} D_{R L}^{\alpha_{1}} e(t), \tau_{0}=\left(E_{1} \tau_{1}^{\alpha_{1}} / E_{2} \tau_{2}^{\alpha_{2}}\right)^{\frac{1}{\alpha_{1}-\alpha_{2}}}, E_{0}=E_{1}\left(\tau_{1} / \tau_{0}\right)^{\alpha_{1}}, \\
\sigma_{m+2}(t)=E_{m+2} \tau_{m+2}^{\alpha_{m+2}} D_{R L}^{\alpha_{m+2}} e(t)(m=1, \cdots, n)
\end{gathered}
$$

and, by this way, we obtain the stress-strain relation given by the fractional order differential equation

$$
\sigma(t)+\tau^{\alpha_{1}-\alpha_{2}} D_{R L}^{\alpha_{1}-\alpha_{2}} \sigma(t)=E_{0} \tau^{\alpha_{1}} D_{R L}^{\alpha_{1}} e(t)+\sum_{j=1}^{n}\left(E_{j+2}^{+} \tau^{\alpha_{j+2}} D_{R L}^{\alpha_{j+2}} e(t)+E_{j+2}^{+} \tau^{\alpha_{j+2}+\alpha_{1}-\alpha_{2}} D_{R L}^{\alpha_{j+2}+\alpha_{1}-\alpha_{2}} e(t)\right)
$$

where $\sigma(t)=\sigma_{0}(t)+\sum_{j=1}^{n} \sigma_{j+2}(t)$ and $\tau=\tau_{0}, E_{j+2}^{+}=E_{j+2}\left(\frac{\tau_{j+2}}{\tau}\right)^{\alpha_{j+2}}, j=1, \ldots, n$.

In Laplace domain, equation (1) reads

$$
\left(1+(s \tau)^{\alpha_{1}-\alpha_{2}}\right) \mathscr{L}\{\sigma(t)\}=\mathscr{L}\left\{E_{0} \tau^{\alpha_{1}} D_{R L}^{\alpha_{1}} e(t)+\sum_{j=1}^{n}\left(E_{j+2}^{+} \tau^{\alpha_{j+2}} D_{R L}^{\alpha_{j+2}} e(t)+E_{j+2}^{+} \tau^{\alpha_{j+2}+\alpha_{1}-\alpha_{2}} D_{R L}^{\alpha_{j+2}+\alpha_{1}-\alpha_{2}} e(t)\right)\right\}
$$

where, taking into account that all quantities are zero for $t \leq 0$, it becomes

$$
\mathscr{L}\{\sigma(t)\}=\frac{E_{0} \tau^{\alpha_{1}} s^{\alpha_{1}} \mathscr{L}\{e(t)\}}{1+s^{\alpha_{1}-\alpha_{2}} \tau^{\alpha_{1}-\alpha_{2}}}+\sum_{j=1}^{n} \frac{E_{j+2}^{+} \tau^{\alpha_{j+2}} s^{\alpha}+E_{j+2}^{+} \tau^{\alpha_{j+2}+\alpha_{1}-\alpha_{2}} s^{\alpha_{j+2}+\alpha_{1}-\alpha_{2}}}{1+s^{\alpha_{1}-\alpha_{2}} \tau^{\alpha_{1}-\alpha_{2}}} \mathscr{L}\{e(t)\} .
$$

Replacing $s$ by $i \omega$, we obtain the complex modulus

$$
G^{*}(\omega)=\frac{\mathscr{L}\{\sigma(t)\}}{\mathscr{L}\{e(t)\}}=\frac{E_{0}(i \omega \tau)^{\alpha_{1}}}{1+(i \omega \tau)^{\alpha_{1}-\alpha_{2}}}+\sum_{j=1}^{n} E_{j+2}^{+}(i \omega \tau)^{\alpha_{j+2}}=G^{\prime}(\omega)+i G^{\prime \prime}(\omega),
$$

where the storage modulus, $G^{\prime}(\omega)$, and loss modulus, $G^{\prime \prime}(\omega)$, are the real and imaginary component of the complex modulus, respectively. Hence, from (2), we have

$$
\begin{aligned}
& G^{\prime}(\omega)=\mathfrak{R}\left(G^{*}\right):=\frac{E_{0}\left[(\omega \tau)^{\alpha_{1}} \cos \frac{\pi \alpha_{1}}{2}+(\omega \tau)^{2 \alpha_{1}-\alpha_{2}} \cos \left(\frac{\pi \alpha_{2}}{2}\right)\right]}{1+(\omega \tau)^{2\left(\alpha_{1}-\alpha_{2}\right)}+2(\omega \tau)^{\alpha_{1}-\alpha_{2}} \cos \frac{\pi\left(\alpha_{1}-\alpha_{2}\right)}{2}}+\sum_{j=1}^{n} E_{j+2}^{+}(\omega \tau)^{\alpha_{j+2}} \cos \left(\frac{\pi \alpha_{j+2}}{2}\right) \\
& G^{\prime \prime}(\omega)=\mathfrak{I}\left(G^{*}\right):=\frac{E_{0}\left[(\omega \tau)^{2 \alpha_{1}-\alpha_{2}} \sin \left(\frac{\pi \alpha_{2}}{2}\right)+(\omega \tau)^{\alpha_{1}} \sin \frac{\pi \alpha_{1}}{2}\right]}{1+(\omega \tau)^{2\left(\alpha_{1}-\alpha_{2}\right)}+2(\omega \tau)^{\alpha_{1}-\alpha_{2}} \cos \frac{\pi\left(\alpha_{1}-\alpha_{2}\right)}{2}}+\sum_{j=1}^{n} E_{j+2}^{+}(\omega \tau)^{\alpha_{j+2}} \sin \left(\frac{\pi \alpha_{j+2}}{2}\right)
\end{aligned}
$$

We note that the Storage and Loss moduli of the FMM are given by the first terms of (3) and (4), respectively.

\section{NUMERICAL OPTIMIZATION AND CONCLUSIONS}

To estimate the $2 n+4$ parameters of the MFZM, complex modulus (2), the storage function (3) and loss function (4) are fitted to the experimental storage $\widehat{G}_{i}^{\prime}$ and loss ${\widehat{G^{\prime \prime}}}_{i}$ moduli data at $N$ frequencies $\omega_{i}$. Thus, the order of the fractional derivatives $\left(\alpha_{1}, \alpha_{2}, \alpha_{3}, \cdots, \alpha_{n+2}\right)$, the spring constants $\left(E_{0}, E_{3}^{+}, \cdots, E_{n+2}^{+}\right)$and relaxation time $\tau$ are determined by solving the nonlinear constrained least squares problem:

$$
\min _{p \in \mathbf{R}^{2 n+4}} F(p) \equiv \sum_{i=1}^{N}\left(\left(\frac{G^{\prime}\left(\omega_{i}\right)}{\widehat{G}_{i}^{\prime}}-1\right)^{2}+\left(\frac{G^{\prime \prime}\left(\omega_{i}\right)}{{\widehat{G^{\prime \prime}}}_{i}}-1\right)^{2}\right)
$$


subject to

$$
\begin{aligned}
& 0 \leq \alpha_{2}<\alpha_{1}<1 \\
& 0 \leq \alpha_{i}<1, \quad i=3, \cdots n+2, \\
& E_{0}>0, E_{i}^{+}>0, \quad i=3, \cdots n+2
\end{aligned}
$$

where $N$ is number of experimental data, $p=\left(\alpha_{1}, \alpha_{2}, \alpha_{3}, \cdots, \alpha_{n+2}, \tau, E_{0}, E_{3}^{+}, \cdots, E_{n+2}^{+}\right)$is the vector of the parameters to be determined, and $G^{\prime}\left(\omega_{i}\right)$ and $G^{\prime \prime}\left(\omega_{i}\right)$ are the predicted values calculated from functions (3) and (4), respectively.

To solve (5) we used the global optimization solver LINDOGlobal (LINDO Systems Global Solver), available online on the NEOS servers for optimization (http: / / neos.mcs. anl.gov/neos/solvers).

The LINDOGlobal solver requires that the optimization problem (5) be coded in GAMS (General Algebraic Modeling System that is a high-level modeling system for mathematical programming and optimization).

The LINDOGlobal finds guaranteed globally optimal solutions to general constrained nonlinear optimization problems containing both continuous and integer decision variables. The LINDOGlobal procedure relies on branch-and-cut techniques [6].

In the experiments, we test the influence of the number of the springpot (fractional) elements in parallel with the classic FMM, giving rise to the MFZM, on the residual value $F$. In Table 1, we report in column 1 the number of springpot considered, column 2 has the number of parameters to be estimated $(2 n+4)$, and column 3 reports the optimal residual value $F^{*}$. In the experiments, as we can see from column 1, we solve the optimization problem (5) considering zero, one, two, three and eight fractional elements. We note that in (5), the storage and loss moduli are fitted to the FMM and FZM models when $n=0$ and $n=1$, respectively.

TABLE 1. Results obtained by LINDOGlobal

\begin{tabular}{ccc} 
n springpot & number of parameters & $F^{*}$ \\
\hline 0 & 4 & 38.36614808 \\
1 & 6 & 1.40236723 \\
2 & 8 & 1.40236723 \\
3 & 10 & 1.33290830 \\
8 & 20 & 1.33290830 \\
\hline
\end{tabular}

As we can see from the Table 1, the residual value is large when the storage and loss functions of the FMM are considered. The residual value decreases rapidly for a small value when FZM is used. When (5) involves the functions of the MFZM, the optimal residual has improved a little for values of $n$ greater than 2 comparatively to the FZM. For values of $n$ greater than 3 it is not possible to improve the optimal residual. Comparing the three models, the MFZM presents the best result. We report in Table 2 the global optimal parameter set of MFZM for $n=3$.

TABLE 2. Optimal parameter set for MFZM $G^{\prime}(\omega)$ and $G^{\prime \prime}(\omega)$.

\begin{tabular}{cccccccccc}
\hline$\alpha_{1}$ & $\alpha_{2}$ & $\alpha_{3}$ & $\alpha_{4}$ & $\alpha_{5}$ & $E_{3}^{+}$ & $E_{4}^{+}$ & $E_{5}^{+}$ & $E$ & $\tau$ \\
\hline 0.7209 & 0.0081 & 0.1004 & 0.0055 & 0.1004 & 50.4212 & $3.2307 \mathrm{E}+3$ & 19.1590 & $1.5156 \mathrm{E}+4$ & $8.3261 \mathrm{E}+7$ \\
\hline
\end{tabular}

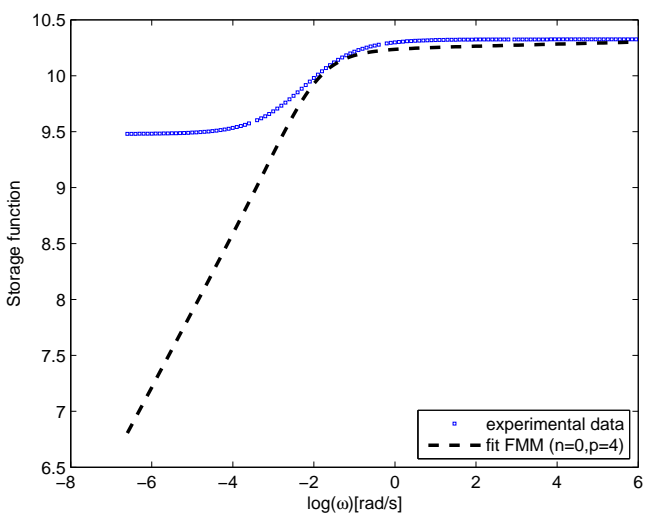

(a) Storage modulus

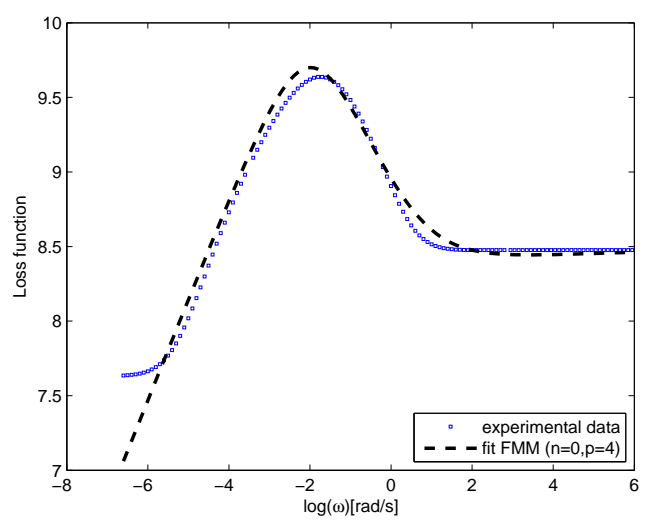

(b) Loss modulus

FIGURE 1. The best fit obtained for the Storage and Loss modulus of the FMM. 


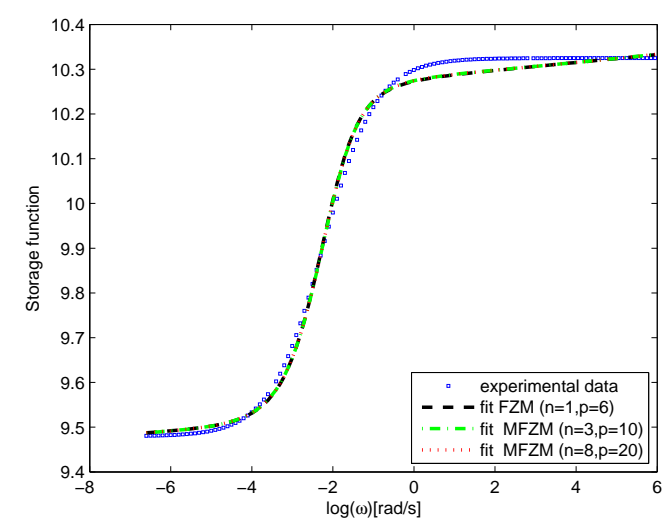

(a) Storage modulus

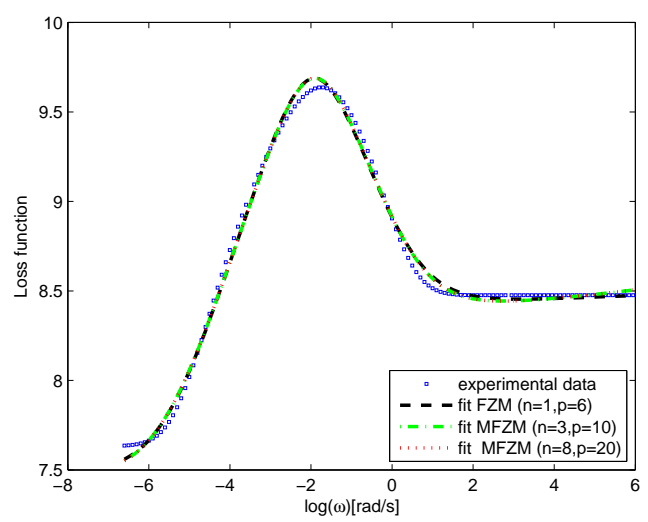

(b) Loss modulus

FIGURE 2. The best fit obtained for the Storage and Loss modulus of the MFZM.

In Figure 1(a) and 1(b) we show the plots of the storage and loss experimental data vs curve-fit of the Storage and Loss functions, respectively, of the FMM. Figure 1 shows that for lower frequencies the Loss an Storage moduli does not reproduce with accuracy the experimental data. These disadvantages are overcome by the Storage and Loss functions of the FZM and MFZM, respectively, which leads to more stable models as we can see in Figure 2.

\section{ACKNOWLEDGMENTS}

This work was financed by FEDER funds through COMPETE (Operational Programme Thematic Factors of Competitiveness) and by portuguese funds through FCT (Foundation for Science and Technology) within the project PEstC/MAT/UI0013/ 2011. We thank the Critical Materials S.A. for making available the DMA experimental data.

\section{REFERENCES}

1. K. Tomas, "Fractional Generalization of the Classical Viscoelasticity Model," Journal of Applied Mathematics 2, pp. 209-216, (2009).

2. I. Podlubny, Fractional Differential Equations. An Introduction to Fractional Derivatives, Fractional Differential Equations, Some Methods of Their Solution and Some of Their Applications, Academic Press, San Diego-Boston-New York-LondonTokyo-Toronto, 1999.

3. M. Fernanda P. Costa and C. Ribeiro, "Parameter Estimation of Viscoelastic Materials: A Test Case with Different Optimization Strategies,"in 9th International Conference of Numerical Analysis and Applied Mathematics, edited by Simos, TE, AIP Conf. Proc. 1389, 2011, pp. 771-774.

4. M. Fernanda P. Costa and C. Ribeiro, "Generalized Fractional Maxwell Model: Parameter Estimation of a Viscoelastic Material" in 10th International Conference of Numerical Analysis and Applied Mathematics, edited by Theodore E. Simos, George Psihoyios, Ch. Tsitouras, Zacharias Anastassi (Eds.), AIP Conf. Proc. 1479, 2012, pp. 790-793.

5. J. G. Liu and M. Y. Xu, "Higher-order fractional constitutive equations of viscoelastic materials involving three different parameters and their relaxation and creep function", Mechanics of Time-Dependent Materials, 10, 2006, pp.263-279

6. Y. Lin and L. Schrage, "The global solver in the LINDO API," Optim. Methods Softw. 24, pp. 657-668, (2009). 